\title{
Preventable warfarin-induced birth defects: A missed opportunity?
}

\author{
M Conradie, ${ }^{1} \mathrm{MB}$ ChB, DCH, FCMG (SA), MMed (Med Gen); B D Henderson, ${ }^{1}$ MB ChB, DCH, MMed (Paed); \\ C van Wyk, ${ }^{2} \mathrm{MSc}$ (Med) Gen Counselling, PhD (HPE) \\ ${ }^{1}$ Division Clinical Genetics, Department of Neurology, Faculty of Health Sciences, University of the Free State, Bloemfontein, South Africa \\ ${ }^{2}$ Division Health Sciences Education, Faculty of Health Sciences, University of the Free State, Bloemfontein, South Africa
}

Corresponding author: M Conradie (conradiemaureen@gmail.com)

\begin{abstract}
Background. Congenital abnormalities and pregnancy losses due to the teratogenic effects of warfarin are prevalent among the South African population. These are potentially preventable if the challenges and barriers faced by at-risk women are understood and addressed effectively. Objectives. To determine the practice, knowledge and attitudes regarding the teratogenic risks experienced by women administered warfarin. Methods. A descriptive study was performed. Quantitative data were collected through a researcher-administered questionnaire. The target population comprised 101 women of reproductive age who received warfarin treatment and attended a single tertiary-level anticoagulation clinic. Results. Patient-related challenges identified in this study are: language barriers, poor understanding of basic terminology and mathematics, poor contraceptive and family planning practices, lack of knowledge regarding the risks of warfarin in pregnancy and passive attitudes towards information attainment.

Conclusions. Interventions are necessary to address the challenges in such settings. These include increased awareness of the teratogenic potential of specific chronic medications among healthcare providers, patients and the public. Standardised management protocols for women of reproductive age initiated on teratogenic medications should be implemented, including contraceptive and family planning discussions at follow-up visits. Improvement of the counselling skills of healthcare providers and the availability of translators or healthcare providers fluent in local languages could assist in risk reduction.
\end{abstract}

S Afr Med J 2019;109(6):415-420. DOI:10.7196/SAMJ.2019.v109i6.13294

Congenital disorders or birth defects are structural or functional anomalies that occur during intrauterine life and can be identified prenatally, at birth or later in life. In $\sim 50 \%$ of all congenital anomalies, no specific cause can be identified. ${ }^{[1]}$

A teratogen is any substance, agent or process that affects typical fetal development, resulting in one or more congenital disorders in the fetus. The type of teratogen, its mode of action, the embryonic process affected, the genetic predisposition, and the stage of development at the time of exposure determine the type and severity of the defect. From approximately the 3rd to the 12th week of gestation - the period during which differentiation of the major organs and systems occurs - the developing embryo is most vulnerable. Known teratogens include chemical and pharmaceutical agents such as warfarin, thalidomide and alcohol; infectious agents, especially rubella, cytomegalovirus and more recently zika virus; ionising radiation; and chronic diseases such as diabetes mellitus. Other risk factors for birth defects include environmental and personal factors, such as age, general health and nutritional status of the mother, or intrauterine trauma to the developing fetus. ${ }^{[1,2]}$

The US Food and Drug Administration (FDA) has a risk category classification system to indicate the level of safety of medication in pregnancy. Medication is rated from A to D according to the level of safety in pregnancy and the level of supporting evidence. For example, medication in category A is completely safe in pregnancy, while that in category $\mathrm{D}$ has proved to have risks in humans and should be avoided, unless the benefit for the mother is greater than the expected risk. Medication in category $\mathrm{X}$ is contraindicated in pregnant women or women who may become pregnant, because it is known to cause birth defects in animals and humans. ${ }^{[3]}$

According to the March of Dimes global report on birth defects,
$2006,{ }^{[4]}$ middle- and low-income countries have a higher incidence of birth defects related to teratogenic medication. This might be owing to the regulation of some of these medications being less strict and to easy over-the-counter availability. Multiple drug use is common, the awareness of the teratogenic potential of certain medications is lacking and many women are unaware of their pregnancy during the first few weeks. ${ }^{[4]}$ In a South African (SA) study, ${ }^{[5]}$ modelled data of genetic causes and an estimate of teratogenic causes showed that at least 1 of 15 children born (6.8\%) is affected by congenital disorders or birth defects. In $19.5 \%$ of these cases, teratogens could be identified as the causative factor. ${ }^{[5]}$

Warfarin, a vitamin K inhibitor, is an anticoagulant used in patients with an increased risk of thromboembolic events. Indications include prosthetic heart valve replacements, some cases of inherited or acquired thrombophilias and cardiac arrhythmias, and the treatment and prevention of recurrent venous thromboembolism (deep-venous thrombosis, pulmonary embolism). ${ }^{[6]}$ In SA, there is a high burden of cardiac abnormalities among young women, mostly due to rheumatic heart disease, which requires replacement of the damaged valves with prosthetic cardiac valves and necessitates life-long anticoagulation therapy. ${ }^{[7,8]}$

When used during pregnancy, warfarin crosses the placental barrier and may cause teratogenic complications, including congenital defects. ${ }^{[7]}$ Therefore, it is classified as category $\mathrm{X}$ in the FDA risk classification system. Warfarin-induced embryopathy has a $>25 \%$ risk of fetal abnormalities, especially during weeks 6 - 9 of gestation. It may also result in central nervous system abnormalities in the 2nd and 3rd trimester, possibly due to microhaemorrhages. Fetal warfarin syndrome consists of nasal hypoplasia, microphthalmia, hypoplasia of the extremities, intrauterine growth retardation, cardiac anomalies, 
scoliosis, deafness and mental retardation. ${ }^{[10]}$ Furthermore, the risk of miscarriages or stillbirths in pregnant women exposed to warfarin increases significantly. ${ }^{[7,9,10]}$ Despite these risks, women with chronic conditions that necessitate anticoagulation would put their own health and life at risk by discontinuing their treatment prior to or during a pregnancy. Ideally, they need to plan pregnancies and switch to low-molecular-weight heparin (LMWH) before the 6th week of pregnancy. In pregnant women at higher risk of thromboembolism, such as those with certain mechanical valve replacements or a history of thromboembolism when receiving heparin, warfarin should be continued in the interest of the mother's health throughout her pregnancy, with replacement by unfractionated heparin or LMWH close to delivery. ${ }^{[1-13]} \mathrm{A}$ limited number of studies have investigated the perspectives and practices of women exposed to potentially teratogenic substances to identify high-risk behaviour and attitudes that could contribute to congenital abnormalities due to teratogenesis. ${ }^{[14-16]}$ International studies have evaluated patients' knowledge and practice with regard to their warfarin treatment in terms of side-effects of bleeding, precautions, monitoring of levels and emergency management. A few standardised questionnaires were verified to test patients' knowledge regarding these aspects. ${ }^{[17,18]}$ No questions were included about the negative effects of warfarin use during pregnancy in terms of birth defects. A local study among women in Johannesburg using warfarin found that they lacked knowledge about the effects of warfarin in general, its effects in pregnancy, the need for planned pregnancies and the management options available. ${ }^{[19]}$

Understanding the challenges and barriers that play a role in teratogenesis and addressing these effectively are necessary to reduce the incidence of drug-related congenital disorders. Poor pregnancy outcomes - presenting as either congenital abnormalities, spontaneous abortion or stillbirth - due to the effects of warfarin, are seen regularly in SA. ${ }^{[8,19]}$ However, epidemiological data and statistics on the exact incidence are not available.

The objective of this study was to determine the knowledge, attitudes and practices of women of reproductive potential regarding the risk of warfarin in pregnancy. The findings may serve to highlight the specific challenges in SA settings and contribute to providing recommendations to address this matter.

\section{Methods}

In this descriptive study, quantitative data were collected by means of a researcher-administered questionnaire. Women of reproductive age, defined as between the ages of 15 and 49 years, currently receiving warfarin treatment, were included in the study. Participants not able to give consent or converse in Sesotho, English or Afrikaans were excluded. No pregnant women were included in this study. Convenience sampling was used by approaching those who attended a specialist anticoagulation clinic in a tertiary hospital. The study was explained by the researchers, a written information sheet was provided, written consent was obtained and data were collected. Data collection was conducted over 8 weeks. This time period was selected because of the frequency of follow-up and to allow enough time to approach as many clinic attendees as possible. There was no limit on the number of participants. Participation was voluntary and anonymous.

The questionnaire focused on the following aspects: (i) demographic information; (ii) details about the initiation of warfarin; information about gravidity, parity and pregnancy outcomes; relationship status, sexual activity and practice with regard to contraception; (iii) patients' knowledge of contraceptives and the teratogenic potential of warfarin; and (iv) attitudes and opinions of women regarding various aspects of teratogenic medication and methods of information delivery.

Information pamphlets in a sealed envelope were given to participants after completion of the questionnaire. The purpose of these pamphlets was to provide education on the teratogenic effects of warfarin and advice on appropriate contraceptive measures and family planning.

Statistical analysis was done by the Department of Biostatistics, University of the Free State (UFS), Bloemfontein, SA. Descriptive statistics, i.e. means and standard deviations or medians and percentiles for continuous data, and frequencies and percentages for categorical data, were calculated.

\section{Ethical approval}

Ethical approval for the study was obtained from the Health Sciences Research Ethics Committee of the Faculty of Health Sciences, UFS (ref. no. HSREC 205/2015) and the Free State Department of Health.

\section{Results}

\section{Demographic information}

A total of 101 women participated in the study. The median age of participants was 34 (range 18 - 49) years. The participants resided in urban and rural areas of Free State Province. Their information with regard to highest level of education, relationship status, employment and home language is summarised in Table 1. Most participants $(n=65 / 98 ; 66.3 \%)$ received secondary education to Grade $10-12$ level. Fifty-one women $(50.5 \%)$ indicated that they were in a casual or full-time relationship. In approximately one-third of participants ( $n=33 ; 32.7 \%)$, their sole source of income was government subsidies. Sesotho was the home language of 63 participants (62.4\%).

\section{Practice}

Table 1. Participants' demographic information regarding level

of education, relationship status, employment, income and home language

\begin{tabular}{|c|c|}
\hline Variable & $n(\%)$ \\
\hline \multicolumn{2}{|l|}{ Level of education $(n=98)$} \\
\hline Grade 5 or lower & $10(10.2)$ \\
\hline Grade 8 or 9 & $12(12.2)$ \\
\hline Grade $10-12$ & $65(66.3)$ \\
\hline Tertiary education & $11(11.2)$ \\
\hline \multicolumn{2}{|l|}{ Relationship status $(N=101)$} \\
\hline Single, separated or widowed & $50(49.5)$ \\
\hline In a casual or full-time relationship & $51(50.5)$ \\
\hline \multicolumn{2}{|l|}{ Employment status $(N=101)$} \\
\hline Unemployed & $76(75.3)$ \\
\hline Casually employed & $17(16.8)$ \\
\hline Permanently employed & $8(7.9)$ \\
\hline \multicolumn{2}{|l|}{ Main source of income $(N=101)$} \\
\hline Government subsidy only & $33(32.7)$ \\
\hline Own income & $26(25.7)$ \\
\hline Partner's income & $22(21.8)$ \\
\hline Family support & $20(19.8)$ \\
\hline \multicolumn{2}{|l|}{ Home language $(N=101)$} \\
\hline Sesotho & $63(62.4)$ \\
\hline Afrikaans & $16(15.8)$ \\
\hline Setswana & $13(12.9)$ \\
\hline isiXhosa/isiZulu & $7(6.9)$ \\
\hline English & $2(2.0)$ \\
\hline
\end{tabular}


The most common indications for warfarin use were heart valve replacements $(n=44$; $43.6 \%)$ and deep-venous thrombosis $(n=43$; $42.6 \%$ ). The duration of treatment ranged from 1 week to 32 years, with a median duration of 4 years. In 37 participants (36.6\%), warfarin was initiated by adult cardiology departments at tertiary level, and in 25 cases $(24.8 \%)$, by general medicine departments at primary care level. Table 2 provides the reason for treatment and level of care where treatment was initiated.

Participants were asked whether they had a pregnancy test prior to the initiation of warfarin; $32(31.7 \%)$ indicated that they were tested and $36(35.6 \%)$ that no pregnancy test was done. Nineteen participants (18.8\%) were unsure, while in 14 (13.9\%) doing a pregnancy test would not have been applicable (3 women were initiated on treatment during pregnancy, 5 were postpartum, 5 were $<15$ years of age, and 1 had undergone tubal ligation). Approximately two-thirds of women (61.3\%) admitted to taking additional chronic medication, of which the most common were unspecified 'cardiac' drugs, antiretroviral and antihypertensive agents.

With regard to sexual activity, 25 participants $(24.8 \%)$ were not sexually active at the time of the study, with the remainder $(n=76 ; 75.2 \%)$ indicating that they were in a sexual relationship. Of the sexually active participants, 15 (19.7\%) were not using any contraception and another 15 (19.8\%) were using male condoms only.

When asked about single v. double contraception, 16 of the 70 women (22.9\%) who used contraception (some used contraception despite not being sexually active at the time of the study) indicated that they were using two different methods of contraception. Eight of the participants (11.4\%) using contraception admitted to not using it regularly. Only 17 of all the participants (17.0\%) reported ever having asked for contraceptive advice, mostly from the nursing sister at the local clinic. A third of the participants $(n=32 / 100 ; 32.0 \%)$ indicated that they were planning future pregnancies.

\section{Outcomes of pregnancies}

Among the 101 women, 87 (86.1\%) had been pregnant at least once. A total of 209 pregnancies were reported by these participants, of which 110 (52.6\%) were planned. Twenty-six of these 87 women (29.9\%) had used warfarin during $\geq 1$ of their pregnancies. The outcomes of 208 of the pregnancies, as reported by the mothers, are indicated in Fig. 1. One live birth was excluded from these calculations, as the relevant child was diagnosed with fetal warfarin syndrome by our department. In all the other cases, pregnancy outcomes were reported by the participants and cases of fetal warfarin syndrome in this group could not be excluded, as it was not known if the children had been specifically evaluated by a geneticist or paediatrician for warfarinrelated birth defects.

\section{Knowledge}

Fewer than half of the women $(n=47 ; 46.5 \%)$ indicated that they had been informed about the effects of warfarin in pregnancy. Of these,

\begin{tabular}{ll} 
Table 2. Details of initiation of warfarin & \\
\hline Initiation of warfarin & $\boldsymbol{n}(\%)$ \\
\hline Indication for warfarin use & $44(43.6)$ \\
Heart valve replacement & $43(42.6)$ \\
Deep-venous thrombosis & $8(7.9)$ \\
Pulmonary embolism & $2(2.0)$ \\
Arrhythmias & $3(3.0)$ \\
Other emboli (central nervous system, cardiac) & $1(1.0)$ \\
Uncertain & \\
Treatment initiated by & $40(39.6)$ \\
Adult cardiologist & $8(7.9)$ \\
Paediatric cardiologist & $37(36.6)$ \\
General medical practitioner & $9(8.9)$ \\
Obstetrician/gynaecologist & $2(2.0)$ \\
Rheumatologist & $2(2.0)$ \\
Neurologist & $1(1.0)$ \\
Endocrinologist & $1(1.0)$ \\
Surgeon & $1(1.0)$ \\
Pulmonologist & \\
Level of care & $26(25.7)$ \\
Primary & $17(16.8)$ \\
Secondary & $58(57.3)$ \\
Tertiary/academic &
\end{tabular}

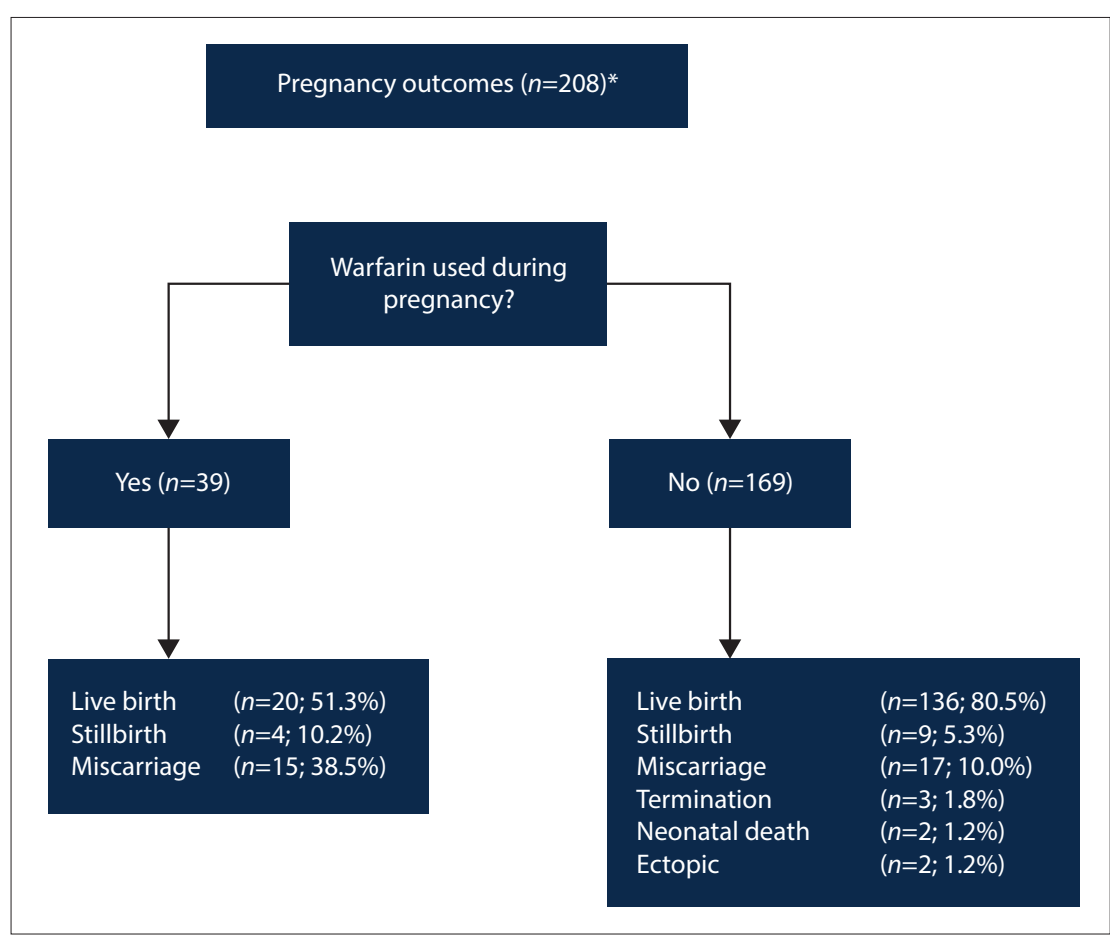

Fig. 1. Pregnancy outcomes in warfarin-exposed $v$. warfarin-unexposed pregnancies. $\left({ }^{*} \mathrm{Of}\right.$ the 101 participants, 87 had been pregnant before, with 209 pregnancies collectively among them. One pregnancy was excluded from the calculations, as the infant was diagnosed with fetal warfarin syndrome.) 
$24(52.2 \%)$ received this information from their doctor and $18(39.1 \%)$ from the nursing sister at the clinic.

The majority of participants $(n=89 / 100 ; 89 \%)$ had no concept or understanding of the term birth defects. An open-ended question about substances that participants might consider to be harmful to a fetus during pregnancy, elicited truly teratogenic substances (e.g. alcohol was listed by 65 participants), but also inappropriate substances or exposures (such as a lack of exercise, stress, lifting heavy objects and using laxatives).

Participants' concept of risk was evaluated. Three different ways of stating the same risk was presented; they had to indicate the highest risk, which included: (i) 10\%; (ii) 1 of 10; and (iii) 10 of 100 . They could also indicate whether all these statements were the same. Option 1 was selected by $39(38.6 \%)$, option 2 by $10(9.9 \%)$ and option 3 by $22(21.8 \%)$ participants. Fewer than one-third $(n=30 ; 29.7 \%)$ of participants indicated correctly that all these expressions of risk were the same.

When asked about the procedure to follow if a pregnancy were desired, the majority ( $n=91 ; 90.1 \%)$ indicated correctly that they should plan the pregnancy and discuss it with the doctor or nursing sister. Four women $(4.0 \%)$ thought they should discontinue their warfarin and $6(5.9 \%)$ that they should discontinue their contraceptives. More than $45 \%$ of the women $(n=47 ; 46.5 \%)$ were of the opinion that the period of highest risk for congenital abnormalities in a pregnancy does not differ between trimesters. Of those who did indicate a specific trimester $(n=54), 39(72.2 \%)$ thought that the first 3 months have the highest risk, while $5(9.3 \%)$ regarded the second trimester as having the highest risk. The remaining participants $(n=10 ; 18.5 \%)$ either indicated the last trimester $(n=9)$ or were uncertain $(n=1)$.

The chance of warfarin causing abnormalities in the baby when administered during pregnancy, was estimated as $5 \%$ by 30 participants $(29.7 \%), 30 \%$ by $17(16.8 \%), 90 \%$ by $22(21.8 \%)$, and $100 \%$ by $29(28.7 \%)$, while $3(3.0 \%)$ were unsure. The women's knowledge of the specific congenital abnormalities associated with warfarin was evaluated. These results are shown in Table 3 . The majority of women either thought that these specific abnormalities could not be caused by warfarin, or were uncertain.

Among the women planning further pregnancies $(n=32)$, knowledge regarding the abovementioned congenital abnormalities was better compared with the group as a whole, with 17 (53.1\%) stating correctly that warfarin could cause brain abnormalities, 9 (28.1\%) skeletal abnormalities and 11 (34.4\%) nasal abnormalities.

The participants' knowledge of the reliability of contraceptive methods in general was assessed by asking which method has the lowest chance of a pregnancy. Oral or injectable contraceptives were indicated by 61 participants $(60.4 \%)$, male and female condoms by $26(25.7 \%)$ and $11(10.9 \%)$, respectively, and checking the calendar by $3(3.0 \%)$.

\section{Attitudes}

Fifty-two participants $(51.5 \%)$ felt that they did not have sufficient information about warfarin in pregnancy, while 38 (37.6\%) indicated that they were satisfied with the amount of information they had, and $11(10.9 \%)$ were unsure. Those who indicated that they did not have sufficient information were asked to provide reasons. According to 27
(52.9\%), they were 'never given the information', 12 (23.5\%) indicated that they 'did not ask for information' and 4 (7.8\%) that they 'did not understand the explanation'. Three participants (5.9\%) said they 'were given information, but forgot because no written material was provided', 2 (3.9\%) 'did not understand the language the information was given in', and the remaining 3 (5.9\%) each gave another reason.

More than two-thirds of women $(n=69 ; 68.3 \%)$ felt that it was their doctors' responsibility to inform them regarding the effects of medication in pregnancy. Twelve (11.9\%) felt it was the responsibility of the clinic nurse and 1 participant (1.0\%) thought it should be the pharmacist's role. Nineteen women $(18.8 \%)$ indicated that all of these healthcare providers should inform patients about the effects of medication taken during pregnancy. Most participants $(n=81 ; 80.2 \%)$ indicated that they would prefer more information about the risks of their medication in pregnancy. The preferred method of information transfer was assessed (Table 4$)$. The majority of participants $(n=74$; $73.3 \%)$ preferred a counselling session that is translated into their home language.

\section{Discussion}

\section{Practice}

Counselling with regard to the potential risks in pregnancy, appropriate contraceptive measures and performing a pregnancy test should form an important part of initiating any potentially teratogenic medication in a woman of reproductive age. Re-evaluating contraceptive options and planning of a pregnancy should ideally form part of every followup consultation in this group of patients, and care should be taken to counsel all girls at the onset of puberty, who had been initiated on teratogenic medication in childhood. ${ }^{[12]}$ It would be beneficial to have a family planning nurse at specialist follow-up clinics, who also understands the complexity of the consultations. For instance, in patients with certain cardiac conditions (such as valve replacements), not only is it important to keep the risks of teratogenesis due to the medication in mind, but also the potential risks for the mother's health during pregnancy and delivery ${ }^{[11]}$ therefore, preventing an unplanned or unwanted pregnancy is all the more important.

Among our study participants, all levels of care, from primary- to academic-level services, as well as professionals in various fields of specialty, were involved in the initiation of warfarin therapy. This emphasises that all healthcare providers involved in different levels of care and in different fields in medicine should be equipped to deal with women of reproductive potential who receive teratogenic medication, or be aware of the need for counselling.

Only a third of participants $(n=32 ; 36.8 \%)$ in whom a pregnancy test prior to initiation would have been indicated, did report it being done. Recall by participants could have been deficient, although further exploration of this observation was beyond the scope of the study.

The number of participants admitting to being sexually active at the time of the study $(n=76 ; 75.2 \%)$ was higher than the number indicating that they were in a casual or full-time relationship $(n=51 ; 50.5 \%)$. This finding could be attributed to short-term sexual encounters that might have resulted in a high risk for unplanned pregnancies and sexually transmitted diseases if effective contraceptive practices were not adhered to. Contraceptive practices were poor, with 30 women (39.5\%) admitting to be sexually active

Table 3. Participants' knowledge about specific abnormalities caused by warfarin $(N=101)$

\begin{tabular}{|c|c|c|c|}
\hline Question & Yes, $n(\%)$ & No, $n(\%)$ & Unsure, $n(\%)$ \\
\hline Can warfarin cause brain abnormalities? & $8(7.9)$ & $32(31.7)$ & $61(60.4)$ \\
\hline Can warfarin cause abnormalities of the bony structure or skeleton? & $9(8.9)$ & $43(42.5)$ & $49(48.5)$ \\
\hline Can warfarin cause abnormalities of the baby's nose, with breathing problems? & $8(7.9)$ & $42(41.6)$ & $51(50.5)$ \\
\hline
\end{tabular}


Table 4. Participants' preference for delivery of information regarding the use of warfarin during pregnancy $(N=101)$ Preferred method of information delivery $n(\%)$ Posters at the clinic $6(5.9)$ Information leaflets in English 2 (1.9) Information leaflets in home language $9(8.9)$ Counselling session in English 5 (4.9) Counselling session translated to home language $74(73.3)$ Pharmacist explaining before handing out medication $\quad 4(3.9)$ Warnings printed on medication box 4 (3.9)

but either not using any contraception or using only male condoms. Additionally, contraception was not used regularly. Effective familyplanning strategies are important to prevent unplanned pregnancies and reduce the risk of babies born with congenital abnormalities. Women who understand the risks and options available will be empowered to implement better contraceptive practices.

Guidelines for managing pregnant patients on anticoagulants for specific indications are available locally and internationally. ${ }^{[11-13,20]}$ The World Health Organization (WHO) medical eligibility criteria for contraceptive use provide specific recommendations for contraceptives in women with chronic medical conditions. ${ }^{[21]}$

\section{Outcomes of pregnancies}

The live birth rate in the pregnancies of women exposed to warfarin was much lower $(n=20 / 39 ; 51.3 \%)$ compared with those not exposed to warfarin $(n=136 / 169 ; 80.5 \%)$. Conversely, the rate of pregnancy loss was much higher in the warfarin-exposed pregnancies due to stillbirths and miscarriages.

According to international figures, $\sim 70 \%$ of pregnancies exposed to warfarin are expected to result in a normal infant, with the remaining $30 \%$ of pregnancies ending in a miscarriage, stillbirth or fetal abnormalities. ${ }^{[10]}$ A Johannesburg study of women with heart valve replacements, who were receiving warfarin, had poor fetal outcomes in $55.2 \%$ of pregnancies. ${ }^{[19]}$ In another study in KwaZulu-Natal, the findings were similar to international figures, with $67 \%$ of warfarinexposed pregnancies resulting in live births. ${ }^{[7]}$ Numerous factors can potentially further contribute to the poorer pregnancy outcomes in our study population. These factors include socioeconomic circumstances, the high burden of infective diseases, inadequate healthcare services for this high-risk group, unequal access to care, and patient factors, such as late presentation to healthcare services and language barriers. ${ }^{[22-27]}$

\section{Knowledge}

The level of education was high in this population group, with 76 women $(77.6 \%)$ having attained at least Grade 10 . This level of education was not in agreement with the participants' poor understanding of basic terminology and mathematics, which was needed to grasp the concept of teratogenesis. Birth defects is a layman's term commonly used in medical practice during the counselling of patients. In the group of women interviewed, $~ 90 \%$ had no understanding of its meaning. Risk is usually expressed as a percentage or a number, e.g. out of 100 or 1000 . Most of the participants $(n=71 ; 70.3 \%)$ had no understanding of these expressions of risk and how to interpret them. Risk is known to be a difficult concept to understand and requires a fair amount of skill and experience to convey during a counselling session. ${ }^{[28]}$

Knowledge regarding the differences in periods of risk for congenital anomalies during a pregnancy was poor. Most participants were also not aware of the specific congenital abnormalities associated with warfarin. This lack of knowledge and understanding about the potentially serious risks of warfarin in pregnancy does not provide a good platform for informed decisions. Their insufficient knowledge could be considered a contributing factor to the poor contraceptive practices and family planning choices that have been observed in this group. A positive observation was that the knowledge of the subgroup of women who were planning a future pregnancy was much better than that of the group as a whole.

The risk of having a child affected by a birth defect due to the use of warfarin during pregnancy was either overestimated $(n=51$; $50.5 \%)$ or underestimated $(n=30 ; 29.7 \%)$. One explanation could be the participants' poor concept of risk. International studies found that healthcare providers and the general public cannot make an accurate estimate of the risks of common medications and substances used during pregnancy. ${ }^{[14,15,29]}$

\section{Attitudes}

We noted a general attitude among the participants of expecting information to be provided without explicitly asking for it. This observation was reflected by the low percentage of women having asked for contraceptive advice, and also by the explanations or reasons provided for their lack of information on the effects of warfarin in pregnancy. Close to $70 \%$ of participants felt that the attending doctor should be responsible for providing this information, suggesting that most participants had a passive attitude rather than active participation in the process of obtaining information.

The majority of women $(n=81 ; 80.2 \%)$ indicated that they would prefer to be more informed about the teratogenic effects of warfarin in pregnancy. It demonstrates their receptiveness to counselling and advice, and opens a window of opportunity to address these issues. Using the correct method of information delivery for the specific target group of patients is very important. The women in our study preferred personal counselling sessions - similar to the opinions of women in a US study ${ }^{[30]}$ More than $70 \%$ of our participants indicated that they would prefer the counselling to be conducted in or translated to their home language.

As part of the study, an information pamphlet was designed, which was distributed to the women after completion of the questionnaire. It included information on the risks of warfarin in pregnancy, alternatives during pregnancy, contraceptive options and contact details for preconception counselling if a pregnancy was desired.

\section{Conclusions and recommendations}

Patient-related challenges that were identified in this study include language barriers, poor understanding of basic terminology and mathematics, poor contraceptive and family planning practices, lack of knowledge regarding the risks of warfarin in pregnancy and passive attitudes towards obtaining information.

Systemic factors were not evaluated, but problems that came to light were the lack of sufficiently skilled counsellors at specialist clinics, lack of contraceptive counselling as part of routine follow-up and lack of interdisciplinary involvement in the care of these highrisk patients.

To decrease the incidence of the potentially avoidable congenital abnormalities and pregnancy losses due to the teratogenic effect of certain medications, these challenges need to be addressed. The matter should be approached with the utmost sensitivity, as there are severe, potentially life-threatening risks to the woman if she discontinues her chronic medication prior to or during a pregnancy, without starting an alternative treatment plan and sufficient monitoring.

Awareness of the teratogenic potential of specific chronic medications 
among all levels of healthcare providers, patients and the public should be improved. We recommend further research to explore the practice, knowledge and attitudes of healthcare professionals involved in initiating women of reproductive age on teratogenic medication. Healthcare providers involved in the care of these women, including nursing staff and pharmacists, have to be aware of and skilled in communicating basic concepts, including risks and uncertainties. This approach requires special training that preferably should commence at undergraduate level. An adequate number of translators and/or healthcare providers fluent in local languages is necessary to overcome the language barriers in the SA healthcare system. Counselling should be done in or translated to the patient's home language where possible, and written material should be available in local languages. This would assist in recall of important aspects of the counselling session and can be kept as a reference.

Specific measures should be put in place to provide improved multidisciplinary care to women of reproductive age who receive potentially teratogenic chronic medication. Clinics where these women are followed up should have contraceptive counselling and family-planning specialists available for consultation. A system could be put in place where potentially teratogenic medication is issued by the pharmacy only after proof of contraceptive counselling/pregnancy testing has been provided. Timely preconception referral to high-risk antenatal clinics should be arranged for these women who desire a pregnancy, to plan alternative treatment strategies during pregnancy and to optimise the mother's health prior to conception. ${ }^{[31]}$ Obstetricians, clinical geneticists and, where appropriate, cardiologists and haematologists should form part of the team at the antenatal clinic.

Adjusting practices and following a person-centred care approach tailored to the needs of our local population, can contribute to reducing the incidence of birth defects and improving the health of mothers with chronic diseases.

\section{Study limitations}

This study was conducted at a single tertiary centre with a specific population profile and included a small sample of participants. The results are not necessarily applicable to other populations at different levels of care or with diverse demographic profiles. Further studies are recommended on a larger population, between multiple centres and among women exposed to various other teratogenic substances.

The women in the study relied on their own recollection when formulating answers that were not confirmed by medical records. Therefore, their feedback might not be an accurate reflection of the services that they received or the outcomes of their pregnancies.

Declaration. This research was conducted by MC in partial fulfilment of her MMed degree in medical genetics.

Acknowledgements. R Nel, Department of Biostatistics, UFS, for assistance with the statistical analysis of data; M E Motloheloa and M E Gono for the collection of data; J Bezuidenhout, head: Division of Health Sciences Education, and A Vorster, clinical psychologist, School of Medicine, UFS, for valuable recommendations; and D Struwig, medical writer/editor, Faculty of Health Sciences, UFS, for technical and editorial preparation of the article. Author contributions. MC was the principal researcher and wrote the initial draft of the article; $\mathrm{BDH}$ and $\mathrm{CvW}$ assisted with conceptualisation, editing and feedback throughout the research process.
Funding. School of Medicine, UFS.

Conflicts of interest. None.

1. World Health Organization. Congenital anomalies. http://www.who.int/mediacentre/factsheets/fs370/ en/ (accessed 15 January 2018)

2. Mosby Inc. Mosby's Dictionary of Medicine, Nursing and Health Professions. 10th ed. St Louis, MO; Elsevier, 2017:1746.
.

3. De Santis M, Straface G, Carducci B, et al. Risk of drug-induced congenital defects. Eur J Obstet Gynecol Reprod Biol 2004;117(1):10-19. https:/doi.org/10.1016/j.ejogrb.2004.04.022

4. Christianson A, Howson C, Modell B. Global report on birth defects: The hidden toll of dying and disabled children. http://www.marchofdimes.org/materials/global-report-on-birth-defects-the-hiddentoll-of-dying-and-disabled-children-executive-summary.pdf (accessed 15 January 2018).

5. Malherbe HL, Christianson AL, Aldous C. Need for services for the care and prevention of congenital disorders in South Africa as the country's epidemiological transition evolves. S Afr Med J 2015;105(3):186188. https://doi.org/10.7196/samj.9136

6. RxList. Clinical pharmacology - prescribing information: Coumadin (warfarin sodium) drug information. http://www.rxlist.com/coumadin-drug/clinical-pharmacology.htm (accessed 15 January 2018).

7. Mazibuko B, Ramnarain H, Moodley J. An audit of pregnant women with prosthetic heart valves at a . Mazibuko B, Ramnarain H, Moodley J. An audit of pregnant women with prosthetic heart valves at a
tertiary hospital in South Africa: A five-year experience. Cardiovasc J Afr 2012;23(4):216-221. https:// tertiary hospital in South Afric
doi.org/10.5830/cvja-2012-022

8. Watkins DA, Sebitloane M, Engel ME, Mayosi BM. The burden of antenatal heart disease in South Africa: A systematic review. BMC Cardiovasc Disord 2012;12:23. https://doi.org/10.1186/1471-2261-12-23

9. Buhimschi CS, Wiener CP. Medications in pregnancy and lactation: Part 1. Teratology. Obstet Gynecol 2009;13(1):166-188. https://doi.org/10.1097/aog.0b013e31818d6788

10. Briggs GG, Freeman RK, Yaffe SJ, eds. Drugs in Pregnancy and Lactation. A Reference Guide to Foetal and Neonatal Risk. 7th ed. Philadelphia, PA: Lippincott Williams \& Wilkins, 2005.

11. Schapkaitz E, Jacobson BF, Manga P, et al. Recommendations for the anticoagulation of pregnant patients with mechanical heart valves. S Afr Med J 2015;105(9):733-738. https://doi.org/10.7196/SAMJnew.7928

12. Royal College of Obstetricians and Gynaecologists. Cardiac disease and pregnancy. Good Practice No.13, June 2011. https://www.rcog.org.uk/globalassets/documents/guidelines/goodpracticel3cardiacdiseaseandpregnancy. pdf (accessed 14 July 2018).

pdf (accessed 14 July 2018).
13. Royal College of Obstetricians and Gynaecologists. Reducing the risk of venous thromboembolism . Royal College of Obstetricians and Gynaecologists. Reducing the risk of venous thromboembolism
during pregnancy and the puerperium. Green-top Guideline No. 37a, April 2015. https://www.rcog.org. uk/globalassets/documents/guidelines/gtg-37a.pdf (accessed 18 July 2018).

14. Sanz E, Gómez-López T, Martínez-Quintas MJ. Perception of teratogenic risk of common medicines. Eur J Obstet Gynecol Reprod Biol 2001;95(1):127-131. https://doi.org/10.1016/s0301-2115(00)00375-4

15. Nordeng H, Ystrøm E, Einarson A. Perception of risk regarding the use of medication and other exposures during pregnancy. Eur J Clin Pharmacol 2010;66(2):207-214. https://doi.org/10.1007/s00228009-0744-2

16. Petersen I, McCrea RL, Lupattelli A, Nordeng H. Women's perception of risks of adverse foetal pregnancy outcomes: A large-scale multinational survey. BMJ Open 2015;5(6):e007390. https://doi.org/10.1136/ bmjopen-2014-007390

17. Winans RM, Rudd KM, Triller D. Assessing anticoagulation knowledge in patients new to warfarin 7. Winans RM, Rudd KM, Triller D. Assessing anticoagulation knowledge in patients new
therapy. Ann Pharmacother 2010;44(7-8):1152-1157. https://doi.org/10.1345/aph.1p092

18. Joshua JK, Kakkar N. Lacunae in patient knowledge about oral anticoagulant treatment: Results of a . Joshua JK, Kakkar N. Lacunae in patient knowledge about oral anticoagulant treatment: Results of a
questionnaire survey. Indian J Hematol Blood Transfus 2015;31(2):275-280. https://doi.org/10.1007/ s12288-014-0415-z

19. Gregersen NE. The implications to women of childbearing age taking warfarin anticoagulation. MSc dissertation. Johannesburg: University of the Witwatersrand, 2005. http://wiredspace.wits.ac.za/ handle/10539/1838 (accessed 15 January 2018)

20. Nishimura RA, Otto CM, Bonow RO, et al. AHA/ACC focused update of the 2014 AHA/ACC guideline for the management of patients with valvular heart disease: A report of the American College of Cardiology/American Heart Association Task Force on Clinical Practice Guidelines. J Am Coll Cardiol 2017;70(2):252-289. https://doi.org/10.1016/j.jacc.2017.03.011

21. World Health Organization. Medical eligibility criteria for contraceptive use. 2015. http://apps.who.int/ iris/bitstream/handle/10665/181468/9789241549158_eng.pdf?sequence=1 (accessed 14 July 2018).

22. Mmusi-Phetoe RMM. Social factors determining maternal and neonatal mortality in South Africa: A Mmusi-Phetoe RMM. Social factors determining maternal and neonatal mortality in South
qualitative study. Curationis 2016;39(1):a1571. https://doi.org/10.4102/curationis.v39i1.1571

23. Wabiri N, Chersich M, Shisana O, Blaauw D, Rees H, Dwane N. Growing inequities in maternal health in South Africa: A comparison of serial national household surveys. BMC Pregnancy Childbirth 2016;16(1):256. https://doi:10.1186/s12884-016-1048-z

24. Amnesty International. Struggle for maternal health: Barriers to antenatal care in South Africa. Amnesty International. 2014. https://www.health-e.org.za/wp-content/uploads/2014/10/Struggle-for-MaternalHealth-pdf (accessed 18 July 2018).

25. Salazar-Austin N, Hoffmann J, Cohn S, et al. Poor obstetric and infant outcomes in human immunodeficiency virus-infected pregnant women with tuberculosis in South Africa: The Tshepiso study. Clin Infect Dis 2018;66(6):921-929. https://doi.org/10.1093/cid/cix851

26. Schoon M, Motlolometsi M. Poor maternal outcomes: A factor of poor professional systems design. Schoon M, Motlolometsi M. Poor maternal outcomes: A factor of
S Afr Med J 2012;102(10):784-786. https://doi:10.7196/SAMJ.6130

27. Govender T, Reddy P, Ghuman S. Obstetric outcomes and antenatal access among adolescent pregnancies in KwaZulu-Natal, South Africa. S Afr Fam Pract 2018;60(1):1-7. https://doi.org/10.1080/20786190.201 7.1333783

28. DeLany Dixon S, Konheim-Kalkstein YL. Risk communication: A complex process. In: LeRoy BS, Veach PM, Bartels DM, eds. Genetic Counseling Practice: Advanced Concepts and Skills. Hoboken, NJ: WileyBlackwell, 2010:65-94.

29. Heaman M, Gupton A, Gregory D. Factors influencing pregnant women's perceptions of risk. MCN Am J Matern Child Nurs 2004;29(2):111-116. https://doi.org/10.1097/00005721-200403000-00010

30. Santucci AK, Gold MA, Akers AY, Borrero S, Schwarz EB. Women's perspectives on counseling about medication-induced birth defects. Birth Defects Res A Clin Mol Teratol 2010;88(1):64-69. https://doi. org $/ 10.1002 / \mathrm{bdra} .20618$

31. Cragan JD, Friedman JM, Holmes LB, Uhl K, Green NS, Riley L. Ensuring the safe and effective use of medications during pregnancy: Planning and prevention through preconception care. Matern Child Health J 2006;10(5 Suppl):S129-S135. https://doi.org/10.1007/s10995-006-0102-2 